Instituto Internacional de Investigación y Desarrollo Tecnológico Educativo INDTEC, C.A.

DOI: https://doi.org/10.29394/Scientific.issn.2542-2987.2020.5.18.14.276-288

OAI-PMH: http://www.indteca.com/ojs/index.php/Revista Scientific/oai

Ensayo Original / Original Essay

\title{
Habilidades gerenciales: Herramientas para fortalecer la cultura organizacional en el ámbito educativo
}

\author{
Autora: Egvani Regían Marín Quero \\ Universidad Pedagógica Experimental Libertador, UPEL \\ egvanirmq3@gmail.com \\ Puerto Cabello, Venezuela \\ https://orcid.org/0000-0002-5953-6358
}

\section{Resumen}

El objetivo del presente ensayo es destacar la importancia de las habilidades gerenciales como herramientas que posee el gerente para el fortalecimiento de la cultura organizacional en el ámbito educativo. Tomando como referencia las Habilidades Técnicas, Humanas, Conceptuales o Estratégicas, haciendo especial énfasis en las habilidades humanas como: la comunicación asertiva, las relaciones interpersonales y el liderazgo gerencial. En Venezuela, las organizaciones educativas centran su atención en una serie de indicadores como: valores sociales, compromiso con sus objetivos y cultura organizacional, donde las herramientas claves para asegurar la eficiencia de los procesos gerenciales son: la mediación entre los intereses del equipo Directivo y las necesidades de los Docentes, la acción directiva eficiente, la creación de espacios de integración a través de la puesta en práctica de estas habilidades. Los fundamentos teóricos y conceptuales se basaron en aportes de autores como Koontz, Weihrich y Cannice (2012); Chaparro (2015); Duarte (2013); Rodríguez (2019); Ramírez (2019); Robbins (2004). Esta investigación de tipo documental resalta la importancia de establecer un ambiente motivador y de integración entre el equipo que gerencia en la Escuela desde la práctica de un estilo gerencial flexible y humanista.

Palabras clave: habilidad; gerente; cultura organizacional.

Cómo citar este ensayo:

Marín, E. (2020). Habilidades gerenciales: Herramientas para fortalecer la cultura organizacional en el ámbito educativo. Revista Scientific, 5(18), 276-288, e-ISSN: 2542-2987. Recuperado de: https://doi.org/10.29394/Scientific.issn.2542-2987.2020.5.18.14.276-288

Fecha de Recepción: 05-06-2020
Fecha de Aceptación: 26-09-2020
Fecha de Publicación: 05-11-2020 


\title{
Management skills: Tools to strengthen the organizational culture in the educational field
}

\begin{abstract}
The objective of this essay is to highlight the importance of managerial skills as tools that the manager possesses to strengthen the organizational culture in the educational field. Taking as reference the Technical, Human, Conceptual or Strategic Skills, with special emphasis on human skills such as: assertive communication, interpersonal relationships and managerial leadership. In Venezuela, educational organizations focus their attention on a series of indicators such as: social values, commitment to their objectives and organizational culture, where the key tools to ensure the efficiency of management processes are: mediation between the interests of the Management team and Teachers' needs, efficient directive action, the creation of integration spaces through the implementation of these skills. The theoretical and conceptual foundations were based on contributions from authors such as Koontz, Weihrich and Cannice (2012); Chaparro (2015); Duarte (2013); Rodríguez (2019); Ramírez (2019); Robbins (2004). This documentary-type research highlights the importance of establishing a motivating and integrating environment among the team that manages the School from the practice of a flexible and humanistic management style.
\end{abstract}

Keywords: skill; manager; organizational culture.

\section{How to cite this essay:}

Marín, E. (2020). Management skills: Tools to strengthen the organizational culture in the educational field. Revista Scientific, 5(18), 276-288, e-ISSN: 2542-2987. Recovered from: https://doi.org/10.29394/Scientific.issn.2542-2987.2020.5.18.14.276-288

Date Received: 05-06-2020
Date Acceptance:

26-09-2020
Date Publication:

05-11-2020 


\section{Introducción}

La presente temática, surge como inquietud personal y profesional de lo observado a diario en el ambiente educativo venezolano, especialmente a nivel gerencial donde los criterios empleados por el personal directivo, obedecen a posturas rígidas que se centran en el poder, tareas y roles asignados a los docentes, perdiendo de vista a la persona como fuerza vital en las organizaciones, limitando así el desarrollo armónico y óptimo de la práctica pedagógica.

Es por ello, que la falta de una efectiva cultura organizacional afecta el buen desenvolvimiento de las actividades dentro de la institución educativa, ocasionando un impacto negativo en el ambiente laboral, creando situaciones de inconformidad, debido a la práctica deficiente o exigua de habilidades gerenciales, como: comunicación asertiva, liderazgo, manejo de las relaciones interpersonales, entre otras, desencadenando en los docentes poca motivación y el desinterés por el trabajo; donde el problema no se centra en el trabajo que se realiza, sino en las relaciones humanas y las actitudes que influyen en los espacios de trabajo.

Esto trae como consecuencia, que se vea afectada la práctica docente, ya que la función de todo gerente es generar condiciones y ambientes de trabajo óptimos que posibiliten el buen desempeño de sus docentes; ahora bien, para lograr el fortalecimiento de la cultura organizacional, el director o gerente de toda institución educativa debe poseer diversas habilidades que le van ayudar a liderar o gestionar de manera eficiente y eficaz el proceso educativo.

Como respuesta a esta realidad es necesario un cambio progresivo de paradigmas y estructuras que se centren en fortalecer la cultura organizacional, desde las habilidades gerenciales, como herramientas de dirección, con una visión y enfoque humanista. Por consiguiente, es necesario que el directivo, no solo conozca estas habilidades gerenciales, sino que 
desde la praxis de las mismas fortalezca la cultura organizacional de su Institución educativa.

\section{Desarrollo}

2.1. Habilidades gerenciales y el fortalecimiento de la cultura organizacional en el ámbito educativo

Las habilidades gerenciales se pueden definir como un conjunto de estrategias, capacidades y conocimiento que debe tener una persona para coordinar, dirigir la fuerza de trabajo en la organización.

Para Koontz, Weihrich y Cannice (2012): las habilidades gerenciales "son un conjunto de capacidades y conocimientos que el emprendedor debe poseer o desarrollar para realizar las actividades de administración, en el rol del Gerente o Director de una organización" (pág. 8). De acuerdo a esto, el docente en su gestión directiva debe ejecutar diversas habilidades, que le ayuden a coordinar y direccionar la Institución educativa, para el alcance de logros organizacionales. Entre ellas se destacan las habilidades técnicas, humanas y las conceptuales o estratégicas.

\subsubsection{Habilidades Técnicas}

Son las conformadas por las capacidades, la pericia y el conocimiento en áreas específicas, las cuales califican a un personal en determinado campo de trabajo. Dichas habilidades se obtienen en el medio académico o de forma empírica a través de la experiencia.

Todo Docente en cargo de Dirección o gerencia debe estar capacitado de manera técnica, además debe estar en constante actualización y formación académica que le aporte de manera oportuna las habilidades y destrezas propias para el desempeño competente y eficaz de su función. 


\subsubsection{Habilidades Humanas}

Las habilidades humanas son esenciales para la práctica de las relaciones interpersonales en el ambiente de trabajo, donde es necesario orientar, inducir, estimular a otras personas sin menoscabo de su nivel, clase, condición, raza, con un enfoque hacia el alcance de las metas organizacionales. Es por ello que tales habilidades son de gran significancia en toda la estructura organizativa, ya que facilitan relaciones positivas entre directivos y subordinados, lo que se puede interpretar como un entorno laboral agradable.

Es de gran relevancia que el equipo que dirige la organización educativa ponga en práctica habilidades humanas o relaciones interpersonales, necesarias para el entendimiento de intereses comunes del personal o recurso humano que integra la Institución, para asegurar el desarrollo de un ambiente favorable y armónico que posibilite el logro de objetivos organizacionales.

\subsubsection{Habilidades Conceptuales o Estratégicas:}

Dichas habilidades tienen que ver con la elaboración e interpretación de nuevos constructos, el desarrollo de estrategias novedosas para la solución de conflictos o situaciones problemáticas que surjan en los espacios de trabajo en momentos determinados.

En el ámbito donde se lleva a cabo el proceso de enseñanza aprendizaje, es común observar cómo se presentan situaciones complejas, que dificultan la toma de decisiones. Es por ello que el Director y su equipo deben estar en la capacidad de desarrollar habilidades estratégicas para dar solución pronta y efectiva a los problemas que se presenten.

\subsection{Las habilidades humanas en el fortalecimiento de la cultura organizacional en el ámbito educativo}

A los efectos de fortalecer la cultura organizacional en el ámbito 
educativo, el gerente debe estar en capacidad de poner en práctica todas las habilidades directivas. Sin embargo, para la presente investigación se hará énfasis en las siguientes habilidades gerenciales humanas: Comunicación asertiva, relaciones interpersonales, estilo o liderazgo gerencial, que desde mi punto de vista, son primordiales en la organización.

Para Román y Fernández (2008), citado por Bermúdez y Bravo (2016): las habilidades directivas "son aquellos comportamientos observables y habituales que posibilitan el éxito de una persona en su función directiva" (pág. 63). La importancia de las habilidades directivas humanas, radican en los hábitos o conductas para gestionar, dirigir y coordinar del Director desde un enfoque humanista, centrado en las personas, donde dicha gestión se base en las necesidades y realidades de la organización educativa. Por tal razón el Gerente debe transformar su rol de burocrático al de un Docente empático, carismático, mediador en lugar de confrontador, capaz de subsanar situaciones de manera armónica, que genere en su personal a cargo bienestar, motivación, todo esto con el fin de alcanzar el éxito y fortalecer la cultura organizacional de la Institución Escolar.

\subsubsection{La comunicación asertiva}

La comunicación asertiva tiene un papel fundamental en la gestión organizacional, ya que a través de la misma se definen las directrices y el canal comunicativo. Es el medio para garantizar el cumplimiento de las mismas, de igual forma es el canal para movilizar el recurso humano de cualquier organización

Por su parte, Rodríguez (2019): afirma que en la actualidad "la comunicación asertiva es un pilar fundamental para garantizar el cumplimiento de los objetivos estratégicos de la organización, es por ello, que se puede considerar la comunicación como un valor estratégico que la gerencia de toda organización debe tener" (pág. 4). 
Atado a lo anterior, es de gran relevancia que el gerente educativo ponga en práctica estas destrezas y habilidades comunicativas, ya que de ello depende en gran medida el éxito o fracaso de su gestión y en consecuencia el de la organización educativa, es por ello que el directivo debe dirigirse al recurso humano que tiene a su cargo: docentes, administrativos, personal de ambiente, y vigilantes; utilizando un lenguaje claro, sin violencia ni posturas rígidas, debe escuchar con empatía, teniendo una visión clara de lo que realmente quiere comunicar al equipo de trabajo.

En el mismo orden de ideas, Chaparro (2015): sostienen que "[...] se conoce como proceso de comunicación asertiva, la expresión clara, directa y equilibrada que cumple con comunicar o defender ideas y sentimientos, sin ningún ánimo o intención de perjudicar al interlocutor(es)" (pág. 8).

La comunicación influye de manera positiva o negativa en las personas que hacen vida en la Institución educativa, cuando se hace uso inadecuado de las habilidades comunicativas no solo por parte del gerente, sino de todo el personal, de allí que posibilita o limita la gestión escolar. Por consiguiente, es de interés que el Directivo comunique de forma, precisa, clara y asertiva lo que realmente desea comunicar a sus Docentes a fin de no crear un clima organizacional tenso y de motivarlos en el alcance de metas fijadas.

\subsubsection{Relaciones interpersonales}

Las relaciones interpersonales son aquellas a través de las cuales se interrelacionan entre si un grupo de personas con intereses comunes, que hacen vida en un entorno idéntico. En concordancia con lo antes descrito, Beiza (2012); y Romero (2016), citados por Ramírez (2019), plantea que:

El ser humano es una pieza fundamental para que dichas relaciones se generen, las mismas se manejan a través de ciertas herramientas que facilitan su uso, tales herramientas son la comunicación, la confianza, la pertenencia, rendimiento, colaboración, entre otros, dependiendo de estos factores para 
que las relaciones interpersonales y todos los tipos de relaciones existentes den como resultado, ambientes afables para los distintos tipos de organizaciones (pág. 12).

En cuanto a lo antes señalado, es de interés para el gerente educativo hacer una revisión crítica y reflexiva de su accionar en torno a las relaciones personales con todos los individuos que forman parte de la organización, para ello debe analizar los procesos internos de comunicación, receptividad, participación, inclusión, empatía, entre otros, que favorezcan la efectividad de relaciones humanas, con el fin de optimizar la cultura organizacional en la Institución Educativa.

De acuerdo con Espinosa (2014): "las auténticas relaciones humanas son aquellas que a pesar de las divergencias lógicas entre las personas, hay un esfuerzo para lograr una atmósfera de comprensión y sincero interés en el bien común" (pág. 25). En base a esto, en las organizaciones escolares la fuerza vital está representada por el personal que la conforma: docentes, administrativos, personal de ambiente y vigilantes; y a su vez la misma será identificada entre una organización y otra, por la calidad en las relaciones interpersonales que exista, así como las actitudes y valores compartidos y de ello dependerá en gran medida el desempeño laboral armonioso y productivo, todos estos aspectos forman parte a su vez de la cultura organizacional.

\subsubsection{Liderazgo gerencial}

Es la capacidad que tiene un gerente de influir de manera positiva y sin ejercer el poder, en la fuerza humana que dirige, para conducirla al alcance de metas y objetivos Institucionales. En línea con lo anterior, Duarte (2013): sostiene que el liderazgo, "es la forma de inspirar confianza entre las personas que componen un equipo y la influencia que se pueda lograr sobre ellas para cumplir las metas establecidas" (pág. 6).

Desde esta perspectiva se hace urgente y necesario que los directivos 
que tienen a su cargo las organizaciones educativas, sean a su vez líderes capaces de ejercer no solo poder y mando Institucional, sino que al mismo tiempo puedan gerenciar con poder humano, desde una visión crítica flexible, carismática y participativa que inspire confianza en los Docentes y a su vez permita generar motivación e inspiración para el desempeño eficiente del rol educativo. En este orden de ideas, Carrero (2019), destaca:

[...] El concepto en si de lo que significa ser un líder se ha ido modificando de acuerdo a los cambios e intereses que presenta la sociedad actual; donde la ética y la responsabilidad como el sentido humano de su accionar, se han convertido en los elementos indispensables para el líder de hoy dentro de las organizaciones [...] (pág. 350).

En relación a lo descrito, se evidencia la necesidad que tiene el gerente educativo de adecuarse a los nuevos retos y paradigmas que exige la sociedad actual, de igual forma, debe adaptarse a la realidad de la organización escolar, desde un estilo del liderar humano y empático que genere en su personal, acciones positivas para el alcance de metas.

\subsection{Importancia de la cultura organizacional en el ámbito educativo}

La cultura organizacional es el sistema de actitudes, valores, hábitos, creencias compartidas que identifican una organización. Por su parte, Robbins (2004): plantea que "la cultura organizacional es un sistema de significados compartidos por los miembros de una organización, que la distinguen de otras" (pág. 525).

Sin embargo, la cultura organizacional se ve afectada por diversos elementos o factores que de una forma u otra va a definir una cultura fuerte, débil o burocrática. Esto determinará a su vez el éxito o fracaso, en relación al cumplimiento de los objetivos organizacionales. No obstante, el directivo debe manejar destrezas y habilidades gerenciales como: el liderazgo positivo, la comunicación asertiva, el buen manejo de las relaciones personales, entre 
otras que son necesarias y de gran significancia para fortalecer dicha cultura organizacional.

De igual modo, la misma debe estar centrada en su personal, ya que el entorno donde se da el proceso de enseñanza-aprendizaje de nuestros niños y adolescentes debe ser un ambiente óptimo, armónico, motivante tanto para los docentes involucrados en la labor educativa, como para el resto del personal que hace vida en la Institución Educativa.

Por lo tanto, en el ámbito educativo venezolano es necesario que el gerente conozca las debilidades de su gestión y las transforme en fortalezas, adopte una cultura organizacional centrada en personas, a través de un liderazgo positivo; así como también, de la práctica de una comunicación asertiva abierta y flexible que genere empatía por el otro.

\section{Conclusiones}

Para finalizar es preciso recordar la importancia que tiene la cultura organizacional en las instituciones educativas venezolanas, ya que contempla una visión holística de su recurso humano, respecto a comportamientos, actitudes, valores compartidos, hábitos, creencias, normas y costumbres, entre otros.

De allí que estos factores a su vez, van a determinar el clima laboral y el grado de cohesión del gerente con su personal, permitiendo de este modo identificar de manera precisa, lo que afecta la cultura organizativa, para la toma de acciones orientadas a fortalecer la misma. Por ello es pertinente la puesta en práctica de ideas innovadoras, donde el director como gerente educativo debe reinventar de forma continua su manera de liderar.

Finalmente, se debe realizar un proceso de revisión constante del equipo que gerencia la escuela, las estrategias, habilidades, formas de planificar y coordinar la fuerza de trabajo representada en el personal docente y demás integrantes que hacen vida en la Institución, ya que de acuerdo al 
grado de bienestar de estos y a la calidad de las relaciones humanas, se podrá determinar si la cultura es débil o fuerte y se verá expresado más tarde en un desempeño laboral eficaz, consustanciado con el proceso de enseñanzaaprendizaje de la calidad que demanda el país en la actualidad.

\section{Referencias}

Bermúdez, E., \& Bravo, V. (2016). Habilidades directivas y desempeño laboral del personal docente en escuelas básicas primarias. Omnia, 22(3). 60-70, e-ISSN: 1315-8856. Recuperado de:

https://www.redalyc.org/articulo.oa?id=73752819005

Carrero, Y. (2019). Identidad Corporativa y los Valores Organizacionales, desde un Liderazgo de Avanzada. Revista Scientific, 4(14), 347-366, e-ISSN: 2542-2987. Recuperado de:

https://doi.org/10.29394/Scientific.issn.2542-2987.2019.4.14.17.347$\underline{366}$

Chaparro, Z. (2015). El uso de la comunicación asertiva como herramienta estratégica para el líder. Trabajo Especial de Grado. Bogotá, Colombia: Universidad Militar Nueva Granada. Recuperado de: http://hdl.handle.net/10654/7868

Duarte, A. (2013). Habilidades Gerenciales y su importancia para el éxito de una organización. Trabajo Especial de Grado. Bogotá, Colombia: Universidad Militar Nueva Granada. Recuperado de: http://hdl.handle.net/10654/10099

Espinosa, M. (2014). Análisis del grado de relación entre las relaciones interpersonales y el clima organizacional percibido por los miembros de la comunidad académica de la Institución Educativa Omaira Sánchez Garzón. Especialización. Cartagena, Colombia: Universidad EAN. Recuperado de: http://hdl.handle.net/10882/8917 Koontz, H., Weihrich, H., \& Cannice, M. (2012). Administración: Una 
perspectiva Global Empresarial. 14ª Edición, ISBN: 978-607-150759-4. México: McGraw-Hill.

Ramírez, L. (2019). Relaciones Interpersonales en el Ámbito Laboral. Trabajo Recepcional. Tlaquepaque, Jalisco: Universidad Jesuita de Guadalajara. Recuperado de:

http://hdl.handle.net/11117/5802

Robbins, S. (2004). Comportamiento Organizacional. 10a Edición, ISBN: 970-26-0423-0. México: Pearson Educación.

Rodríguez, D. (2019). Comunicación Asertiva para la toma de Decisiones Gerenciales. Artículo de investigación. Bogotá, Colombia: Universidad Militar Nueva Granada. Recuperado de:

http://hdl.handle.net/10654/31896 


\section{Egvani Regían Marín Quero}

e-mail: egvanirmq3@gmail.com

Nacida en Puerto Cabello, Venezuela, el 1 mayo del año

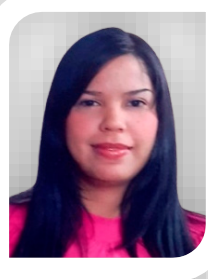
1981. Realicé mis estudios de pregrado en la Universidad de Carabobo (UC), obteniendo el título de Licenciada en Educación, mención Orientación, en el año 2005; y graduada como Abogada, en la Universidad Bolivariana de Venezuela (UBV) en el año 2016; desempeño funciones de orientación y asesoramiento en la Unidad Educativa Integral Colegio José Antonio Maitin en Puerto Cabello, estado Carabobo, Venezuela.

El contenido de este manuscrito se difunde bajo una Licencia de Creative Commons ReconocimientoNoComercial-Compartirlgual 4.0 Internacional 